\title{
THE COSMIC MICROWAVE BACKGROUND AFTER MAXIMA AND BOOMERANG
}

\author{
P. L. RICHARDS \\ Department of Physics, University of California at Berkeley, \\ Berkeley, CA 94720-7300, U.S.A. \\ and Materials Sciences Division, \\ Lawrence Berkeley National Laboratory, Berkeley, California, U.S.A.
}

\begin{abstract}
:
The first release of data from the MAXIMA and BOOMERANG experiments has introduced a new era of precision cosmology. The two data sets are essentially independent, consistent and complementary. In a joint effort by the two teams, the two data sets were combined and then used to test cosmological models and determine values of cosmological constants. These results are available because of the success of bolometric detection techniques. The experimental approach is described with references to the MAXIMA-1 experiment. Important new cosmological experiments at far infrared and millimeter wavelengths require major improvements in bolometric techniques. A new technology, the voltage-biased superconducting bolometer, promises to provide the required experimental power.
\end{abstract}

\section{The MAXIMA-1 Experiment}

Measurements of the anisotropy of the cosmic microwave background (CMB) can discriminate between cosmological models and determine cosmological parameters with high accuracy (Kamionkowski and Koswoski, 1999) [1]. MAXIMA is a balloon borne experiment optimized to map the CMB anisotropy over hundreds of square degrees with an angular resolution of 10 arcmin and to produce measurements of the CMB power spectrum over the range $80<\ldots<800$.

\subsection{INSTRUMENTATION}

Lee, et. al. [2] gives a detailed description of the MAXIMA system. It is an off-axis Gregorian telescope mounted on an attitude controlled balloon platform. The primary mirror rotates so as to scan the telescope beams in azimuth. Re-imaging optics in the receiver cryostat are operated at $\sim 3 \mathrm{~K}$. The receiver consists of 16 photometers: eight operating at $150 \mathrm{GHz}$, four at $240 \mathrm{GHz}$ and four at $410 \mathrm{GHz}$. All have 10' FWHM beams. CMB radiation is detected with spider web bolometers (Bock, et. al. [3]) operated at $0.1 \mathrm{~K}$. The detector time constants, determined in flight, range from 5 to $13 \mathrm{~ms}$. Bolometers are 
AC biased to avoid low frequency amplifier noise. The combined sensitivity of the eight $150 \mathrm{GHz}$ photometers is $41 \mathrm{microK} \infty \mathrm{rt}(\mathrm{sec})$.

The gondola azimuth is driven by a reaction wheel using information from a two-axis magnetometer and three rate gyroscopes. Pointing is reconstructed using a CCD camera bore-sighted to the center of the primary mirror scan and an offset CCD camera that views Polaris. An on-board computer locates the two brightest stars in each CCD field at $5 \mathrm{~Hz}$.

\subsection{OBSERVATIONS}

The instrument was launched from the National Scientific Balloon Facility in Palestine, Texas at 1.6 UT 1998 August 2. The CMB anisotropy was measured in two overlapping observations. Each was conducted at a constant elevation angle and consisted of two independent modulations in azimuth: a slow modulation of the entire instrument $+/-5.6$ deg, $68 \mathrm{sec}, 45 \mathrm{deg}$ elevation for the $1 \mathrm{st}$ scan; +/- $3.3 \mathrm{deg}, 47 \mathrm{sec}, 31$ deg elevation for the 2nd scan) and a fast, total power modulation using the primary mirror $(+/-2 \mathrm{deg}, 0.5 \mathrm{~Hz}$ for both scans). The first observation began at 4.3 UT and lasted 1.7 hours. The second began at 6.0 UT and lasted 1.4 hours. Due to the rotation of the sky, the two scans were cross-linked at an angle of $22 \mathrm{deg}$. The gondola altitude varied from 38.0 to $38.8 \mathrm{~km}$ during the $\mathrm{CMB}$ observation.

\subsection{CALIBRATION}

A full beam calibration of the 150 and $240 \mathrm{GHz}$ photometers was obtained from the CMB dipole. The instrument was rotated 100 times with a period of 20 seconds from 3.6 to 4.2 UT. The data from each rotation was fitted to a model including the CMB dipole and Galactic dust emission (Finkbeiner, et. al. [4]). A component correlated with the $410 \mathrm{GHz}$ channel signal is consistent with atmospheric contribution and was subtracted. Beam contour maps and were obtained from the Jupiter observation from 7.5 to 8.1 UT. The beam profiles were integrated and used with the angular diameter and brightness temperature of Jupiter (Goldin, et. al. [5]) to calibrate the photometers.

\subsection{DATA ANALYSIS}

The raw data for each photometer consisted of 1.2 million samples for the first CMB scan and 1 million samples for the second. The data were deglitched, the electronic and bolometric transfer functions deconvolved, and estimates were made of the noise power spectrum of the data stream. It was assumed that the time domain data were dominated by noise and the procedure of Ferreira and Jaffe [6] were used to confirm the validity of this assumption. High and low pass filters were used to eliminate frequencies where there are no appreciable optical signals.

The calibrated time stream data were combined with the attitude pointing solution to produce a maximum likelihood pixelized map of temperature anisotropy and a pixel-pixel noise correlation matrix using the techniques described in Wright [7], Tegmark [8], Bond et. al. [9], and Ferreira and Jaffe [6]. Individual 100 square degree maps were generated from the outputs of four individual photometers. After checking for consistency and 
verifying that the noise in different maps was uncorrelated, a noise weighted average temperature anisotropy map was then produced. The maximum likelihood angular power spectrum $\mathrm{C}_{\ldots}$ of the map was generated using the MADCAP (Borrill [10]) implementation of the Newton-Raphson iterative maximization of the likelihood function following Bond, Jaffe \& Knox [4].

Analysis of data from the MAXIMA-2 flight, launched in July 1999, has begun. This flight measured over 200 square degrees of the sky. Approximately $25 \%$ of this observing region overlaps with the MAXIMA-1 observations.

\subsection{FOREGROUNDS}

Foreground sources of confusion may include emission from the atmosphere, interstellar dust emission, synchrotron radiation, free-free emission, and point sources. The spectrum of the observed signal is not consistent with that of the atmosphere. The temporal stability of the observed sky structure is inconsistent with an atmospheric or ground-based origin. The ground based telescope sidelobe measurements (Lee, et. al. [2]) also provides evidence that sidelobe response from the ground or the moon is not significant.

The 100 micron IRAS/DIRBE map (Schlegel et. al. [12]) was extrapolated to the MAXIMA wavebands and cross-correlated with the CMB maps. No correlations large enough to effect CMB power spectrum estimation were found. Using the galactic emission model of Bouchet and Gispert [13] it was found that both Bremstrahlung and Synchrotron emission are expected to contribute fluctuations with a magnitude less than 1 microK at $150 \mathrm{GHz}$.

A thorough catalog search did not reveal any radio sources that are likely to produce detectable signal in our maps at $150 \mathrm{GHz}$. No corrections to the data were required for any source of foreground contamination.

\subsection{COSMOLOGICAL PARAMETERS}

The MAXIMA map and power spectrum [14] was released a few weeks after the corresponding announcement from BOOMERANG [15]. The two data sets are essentially independent, consistent and complementary [14]. BOOMERANG has more statistical weight but MAXIMA extends to higher angular frequencies and has a more accurate calibration. In a joint effort by the two teams, the two data sets were combined by marginalizing over differences in calibration and beam uncertainties and then used to test cosmological models and determine values of cosmological constants [16]. The combined power spectrum clearly defines the first acoustic peak and is consistent with a range of flat $\Lambda \mathrm{CDM}$ models with a relatively large baryon fraction. The data are consistent with the second and third acoustic peaks predicted by the models but only if the second peak is somewhat smaller than previously expected [16]. This implies a somewhat larger baryon fraction than that predicted from big bang nucleosynthesis. The data do not demonstrate the existence of a second of third peak because they are also consistent with a horizontal straight line for values of ... above the first acoustic peak. A 
seven parameter Bayesian analysis was used to deduce cosmological parameters. The results support the picture of a flat universe, a flat primordial power spectrum, and a baryon fraction of $\sim 5 \%$. When combined with supernova data to break the degeneracy in the $\Omega_{\Lambda} \Omega_{\mathrm{m}}$ plane, the picture emerges of a universe that is $\sim 70 \%$ dark energy, and $\sim 25 \%$ dark matter. If the supernova data are not considered, and data for large scale structure are used to break the degeneracy, the conclusion remains essentially the same.

There is much interest in the quantitative details of the deduced parameters especially the apparent discrepancy between the values of baryon fractions obtained from CMB and nucleosynthesis. At this early stage, however, the general agreement obtained for the values of total density and the fractions of dark energy, dark matter and baryons should be considered a triumph for experimental cosmology.

\section{The Future of Bolometric CMB Experiments}

At present, CMB experiments are done with two very different types of receiver. High electron mobility transistor (HEMT) amplifiers are phase-conserving linear photon amplifiers whose sensitivity is limited by quantum noise. Bolometers, by contrast, are square-law detectors which do not preserve phase so have no such sensitivity limit. In principle, bolometers can reach the sensitivity limit set by photon statistics. The best photometers using current spider web bolometers, with germanium thermistors and JFET amplifiers [3] operate close to this limit for photon rates observed near the peak of the CMB spectrum. In spite of their limited sensitivity, however, HEMT receivers are used for many current $\mathrm{CMB}$ temperature anisotropy experiments including the interferometers CBI, DASI, and VSI and the MAP and PLANCK LFI orbital missions. Part of the reasons for this preference is that the operating characteristics of HEMT amplifiers are generally superior to those of bolometers. These include dynamic range, linearity, sensitivity to temperature and infrared power loading, speed, sensitivity to rf interference, required operating temperature, reproducibility, etc. These operating limitations make bolometric systems very difficult to optimize and often limit the performance achieved. Both HEMT and bolometer technologies are limited by complexity to relatively small arrays. The interferometers have up to 14 receivers, the best bolometric systems use 16-100 bolometers.

A number of cosmological experiments now under consideration will require much more rapid mapping of the sky than is now possible. These include proposed measurements of the E and B-mode polarization anisotropy of the CMB, statistical studies of the SunyaevZeldovich effect at arcmin resolution in clusters at $Z=1$ and studies of the sources of the far infrared background. For these experiments we need large arrays of $10^{3}-10^{4}$ bolometers with improved operating characteristics.

\section{The Voltage-Biased Superconducting Bolometer}

A new bolometric technology is now being developed which promises to supply the required performance in large format arrays. The voltage-biased superconducting 
bolometer (VSB) $[17,18]$ uses a superconducting thin film transition edge temperature sensor (TES). The voltage bias creates a strong negative electrothermal feedback that keeps the bolometer operating temperature constant despite changes in refrigerator temperature or infrared power loading. The VSB has a large number of advantages over conventional bolometer technology. The feedback increases the bolometer speed and the linearity, and reduces the sensitivity to environmental factors. The Johnson noise is suppressed. The bolometers operate at low impedance, where microphonic effects are reduced, and use superconducting quantum interference (SQUID) amplifiers. These amplifiers dissipate negligible power, operate at low temperatures and have very low noise. Perhaps most important, the VSB is produced entirely by thin film deposition and optical lithography, and so can be fabricated in large format arrays. Also, the large noise margin of the SQUID amplifiers makes it possible to build readout multiplexers which can read out many bolometers through a single amplifier.

\subsection{STATUS OF VSB DEVELOPMENT}

Because of the attractive properties of the VSB, a number of groups are actively pursuing development. Much of the work in the open literature comes from the Berkeley group which will be featured here. Leg-isolated bolometers with round (spiderweb) mesh absorbers have been successfully produced by thin film deposition and optical lithography [19]. Sources of excess noise have been explored and techniques to eliminate them have been found [20]. Excellent low frequency (1/f) noise performance has been demonstrated without ac bias, square format mesh absorber bolometers with compact legs suitable for arrays have been fabricated and tested [21]. Mechanical structures (legs and mesh absorbers) have been fabricated with high yield in a 1024 bolometer close-packed array format [21]. A primitive hot electron (electron-phonon isolated) VSB has been successfully tested [17]. The Berkeley group (and others) are investigating antenna coupled VSB's. The antenna output can be coupled to the bolometer with a superconducting transmission line which can have very low loss at CMB frequencies and bolometer operating temperatures. The advantage of this approach is that the antenna output can be split into a number of bands before detection by transmission line

multiplexers and bandpass filters. This is particularly convenient for CMB measurements where simultaneous measurements at several frequencies is extremely useful.

\subsection{OUTPUT MULTIPLEXERS}

Large format arrays of $10^{3}$ or more bolometers are essentially impossible without output multiplexing. Present technology requires one amplifier in the cryostat for each bolometer. Each amplifier requires $\sim 5$ wires. An array of $10^{3}$ bolometers would then require $5 \infty 10^{3}$ wires entering the cryostat. This is not remotely possible. The technologies used to detect electromagnetic waves at infrared, optical and higher frequencies rely on output multiplexing schemes such as the CCD. The great scientific potential of the millimeter/submillimeter band will not be realized without an analogous output multiplexer scheme. 
The group at the National Institute of Standards and Technology (NIST) was the first to address this critical need. Because the noise in the SQUID amplifier is $\sim 10$ times lower than the typical bolometer output noise, it is in principle possible to read out $\sim 100$ bolometers through a single amplifier. The NIST group chose a switching approach in which SQUID's are used to switch the output of a row of detectors sequentially to a single amplifier [22]. The Berkeley group is exploring a frequency domain multiplexer which ac biases each detector in a row with a different frequency and separates the signals with lock-in amplifiers at the SQUID output [23]. It is not clear at this stage which approach will be best. Both appear to be capable of providing the output multiplexing that is absolutely essential for successful large format bolometer arrays.

\section{Acknowledgments}

This paper summarizes the work of a very large number of people. The discussion of the MAXIMA project draws heavily on a manuscript written by B. Rabii for the MAXIMA team. The paper describing the combined MAXIMA/BOOMERANG parameter extraction has 42 authors [16]. The information on the VSB future bolometric arrays depends extensively on the work of the Berkeley bolometer group.

This research was supported by the National Science Foundation, NASA, the U.S. National Energy Research Scientific Computing Center (DOE), and by the Director, Office of Science, Office of Basic Energy Sciences of the U. S. Department of Energy under Contract No. DE-AC03-76SF00098. 


\section{References}

1. Kamionkowski, M., and Kosowsky, A. (1999) The Cosmic Microwave Background and Particle Physics, Ann. Rev. Nucl. Part. Sci. 49, 77-123, astro-ph/9904108.

2. Lee, A. T., et. al. (1998) MAXIMA: An Experiment to Measure Temperature Anisotropy in the Cosmic Microwave Background, in L. Maiani, F. Melchiorri and N. Vittorio (eds.), AIP Conf. Proc. 476, pp 224-236.Proceedings of $3 \mathrm{~K}$ Cosmology Conference, Rome, Italy, astro-ph/9903249.

3. Bock, J. J., et al., (1995) A Novel Bolometer for Infrared and Millimeter-Wave Astrophysics, Space Sciences Reviews 74, 229-235.

4. Finkbeiner, D. P., et. al. (1999) Extrapolation of Galactic Dust Emission at 100 Microns to CMBR Frequencies Using FIRAS, Ap. J. (in press) astro-ph/9905128

5. Goldin, A. B., et. al. (1997) Whole Disk Observations of Jupiter, Saturn and Mars in Millimeter-Submillimeter Bands, Ap.J.Lett. 488, L161, astro-ph/9612040.

6. Ferreira, P. G., and Jaffe, A. H. (1999) Simultaneous Estimation of Noise and Signal in Cosmic Microwave Background Experiments, MNRAS (to be published) astro$\mathrm{ph} / 9909250$.

7. Wright, E. (1996) Scanning and Mapping Strategies for CMB Experiments, Report UCLA-ASTRO-ELW-96-03, presented at the IAS CMB Data Analysis Workshop in Princeton, astro-ph/9612006.

8. Tegmark M. (1997) How to Make Maps from CMB Data Without Losing Information, Ap. J. 480, 87, astro-ph/9611130.

9. Bond, J. R., et. al., (1999) Computing Challenges of the Cosmic Microwave Background, Computing in Science and Engineering 1, 21.

10. Borrill, J, (1999) MADCAP-The Microwave Anisotropy Dataset Computational Package, Proceedings of the Fifth European SGI/Cray MPP Workshop, astro$\mathrm{ph} / 9911389$.

11. Bond, J. R., Jaffe, A. H., and Knox, L. (1998) Estimating the Power Spectrum of the Cosmic Microwave Background, Phys. Rev. D57, 2117.

12. Schlegel D. J., et. al. (1998) Ap. J. 500, 525, Application of SFD Dust Maps to Galaxy Counts and CMB Experiments, in S. Colombi and Y. Mellier (eds.) Wide Field Surveys in Cosmology, (in press) astro-ph/9809230.

13. Bouchet, R. B., and Gispert, R., (1999) Foregrounds and CMB Experiments: I. SemiAnalytical Estimates of Contamination, New Astron. 4, 443-479 astro-ph/9903176. 
14. Hanany, S., et al., MAXIMA-1: A Measurement of the Cosmic Microwave Background Anisotropy on Angular Scales of 10 Arcminutes to 5 Degrees, Ap. J. Lett. (in press) astro-ph/0005123, .

15. de Bernardis, P., et al. (2000) A Flat Universe from High-Resolution Maps of the Cosmic Microwave Background Radiation, Nature 404, 955-959.

16. Jaffe, A.H., et al. Cosmology from Maxima-1, Boomerang and COBE/DMR CMB Observations, Phys. Rev. Lett. (submitted).

17. Lee, A.T. et al. (1996) A Superconducting Bolometer With Strong Electrothermal Feedback, Appl. Phys. Lett. 69, 1801.

18. Lee, A.T. et al. (1997) Voltage-Biased High-T ${ }_{c}$ Superconducting Infrared Bolometers with Strong Electro Thermal Feedback, IEEE Trans. Appl. Superconductivity 7, 2378

19. Gildemeister, J.M., Lee, A.T., and Richards, P.L., (1999) A Fully Lithographed Voltage-Biased Superconducting Spiderweb Bolometer, Appl. Phys. Lett. 74, 868.

20. Gildemeister, J.M., Lee, A.T., and Richards, P.L., A Model for Excess Noise in Voltage-Biased Superconducting Bolometers, Appl. Optics Lett. (in press).

21. Gildemeister, J.M., Lee, A.T., and Richards, P.L., Monolithic Arrays of AbsorberCoupled Voltage-Biased Superconducting Bolometers, Appl. Phys. Lett. (in press).

22. Chervenak, J.A., et al. (1999) Superconducting Multiplexer for Arrays of Transition Edge Sensors, Appl. Phys. Lett. 74, 4043-4045.

23. Yoon, J., Clarke, J., Gildemeister, J.M., Lee, A.T., Myers, M.J., Richards, P.L., Skidmore, J.T., A Single SQUID Multiplexer for Arrays of Low Temperature Sensors, Appl. Phys. Lett. (in press). 